\title{
Comparison of Quadriceps Muscle Girth Using Ultrasound Imaging In Supervised vs Unsupervised Post Operative ACL Reconstruction
}

\author{
Sumit Saxena ${ }^{1}$, Aparna Sarkar ${ }^{2}$, A.K. Naik ${ }^{3}$ \\ ${ }^{1}$ PhD Scholar, Amity Institute of Physiotherapy, Amity University Uttar Pradesh, India, ${ }^{2}$ Professor Physiology, \\ Amity Institute of Physiotherapy, Amity University Uttar Pradesh, India, ${ }^{3}$ Professor, Department of Orthopedic, Dr. \\ RML Hospital, Delhi, India
}

\begin{abstract}
Injury to the anterior cruciate ligament (ACL) is one of the most common knee ligament injuries. The ACL injury is a sprain or tear of the anterior ligament (ACL). Effective exercises will reduce the risk of ACL injury. An ACL injury in athletes is a higher incidence than other general public, such as soccer, basketball, hockey, etc. The aim of this studywas to compare the effectiveness, recovery rate and variance in both knee-affected and non-affected muscle girth readings in supervised $\mathrm{v} / \mathrm{s}$ unsupervised patient groups after the patient underwent ACL reconstruction surgery. The participants were divided into two groups(A-Supervised and B-Unsupervised) through a random sampling. The supervised exercise in group A performed according to the WILK protocol at RML hospital and un-superviseed group B performed the same WILK protocol at home. The ultrasound scans were performed after 2-4 weeks and 12-14 weeks post operation to measure the muscle girth in both the groups. The study findings indicated the significant difference in recovery rate as well as the difference in between supervisedv/s unsupervised groups i.e. the difference of affected and unaffected knees based on ultrasound imaging was higher in un-supervised group compared to supervised group (a difference of $2.3 \mathrm{~mm}$ in post-operation muscle girth after 12-14 weeks). The ultrasound reading of the muscle girth after 2-4 weeks post-operation showed a decline in the recovery of the muscle girth in both the groups ( $9 \%$ and $8 \%$ for supervised and un-supervised groups respectively). This reduction in the muscle girth value as compared to the pre-operative values was due to arthroscopic effect, which showed there was a transient deactivation of the muscle take place due to surgery. The ultrasound reading of the muscle girth after 12-14 weeks post-operation showed a different progressive recovery of the muscle girth in both the groups as $31 \%$ and $10 \%$ for supervised and un-supervised groups respectively. We concluded that supervised exercises was better than unsupervised exercises for the early recovery after ACL recontraction.
\end{abstract}

Keywords: Quadriceps; Muscle girth; ACL; Ultrasound; Recovery.

\section{Introduction}

The knee is a weight-bearing joint which operates as a fulcrum between tibia and femur. ACL is present in the knee and the bone structure of the knee joint is

\author{
Corresponding Author: \\ Sumit Saxena \\ $\mathrm{PhD}$ Scholar, Amity Institute of Physiotherapy, Amity \\ University Uttar Pradesh, India \\ e-mail: saxena_sumit14@yahoo.co.in
}

made up of the tibia, femur and patella ${ }^{1,2}$. The knee is basically a pivoted joint that is held together by the Posterior Cruciate (PCL) ligaments, Medial Collateral (MCL), Anterior Cruciate (ACL) and Lateral Collateral $(\mathrm{LCL})^{3,4}$. The ACL is located diagonally in the knee as is shown in the below figure, keeping the tibia from sliding out before the femur and also giving rotational steadiness to the knee. The ACL is one of the four primary ligaments inside the knee that associate the femur to the tibia $^{5-7}$. ACL tends to be at risk as it prevents posterior displacement of the distal femur on the tibia. ACL can tear due to twisting type of force acted at the joint in such 
a way that femur and tibia twist in opposite directions. ACL reconstruction may be defined as the functional restoration of the ACL to its native dimensions, collagen orientation and insertion sites. ACL Reconstruction is the technique in which the tunnels are placed in the centre of the native femoral and tibial insertion sites ${ }^{8-10}$. It can be done either through a single bundle or double bundle. Anatomic ACL reconstruction can be used in both single and double-bundle reconstructions and also to Augmentation surgery. Complete restoration of the native ACL may not be possible, because of the complex nature of the ligament. However, the surgeons always attempt towards close proximity ${ }^{11-13}$.

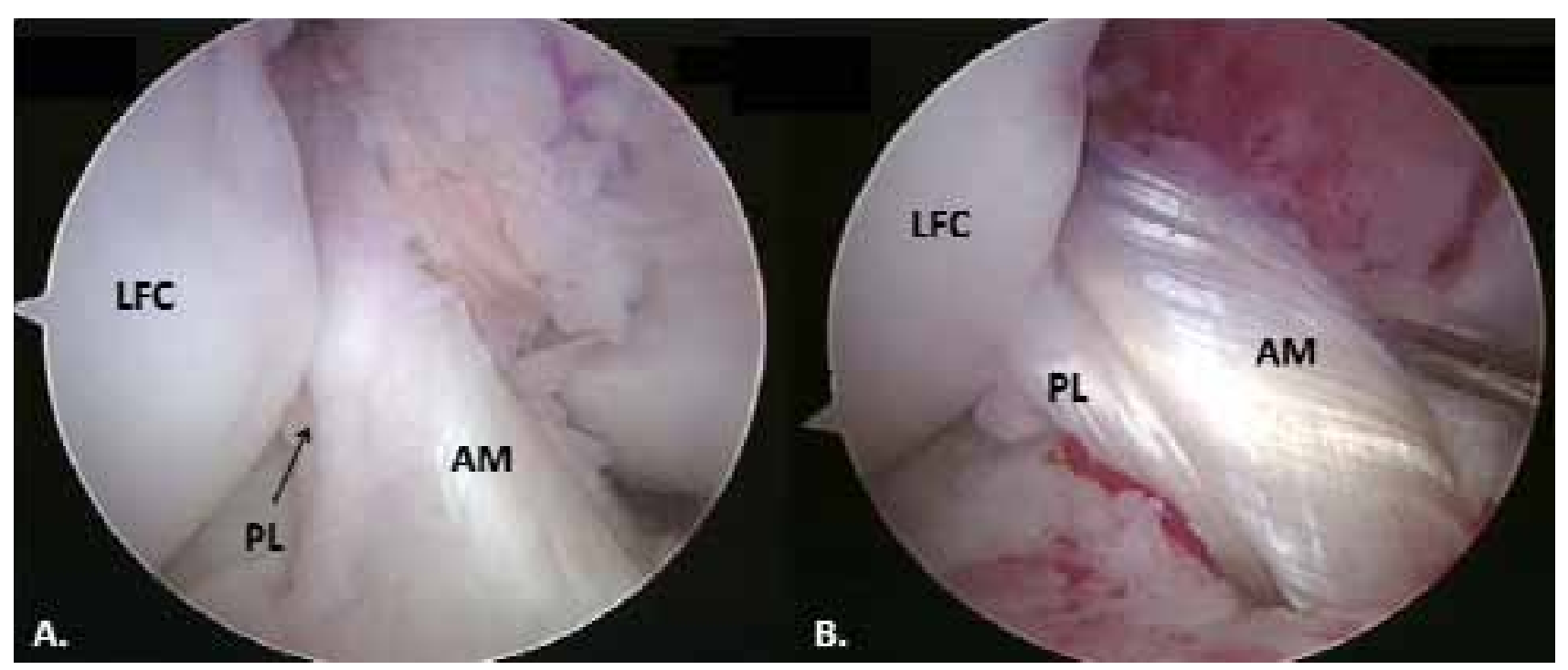

Figure 1. ACL reconstruction restores the native ACL anatomy

ACL injuries received the greater attention of orthopaedic surgeons and due to their deep analysis of the subject, it became possible to treat such injury ${ }^{14,15}$. Previously, the cases of ACL were not reported so frequently in India as in the current scenario due to the absence of sufficient analytical skills $\&$ required tools . But now the incidences of ACL has increased and after an ample amount of research in this field, the surgeons are able to perform ACL Reconstruction successfully. There is as low as $2.5 \%$ chance of recurrent instability due to rupture of the reconstructed ligament or maybe poor surgical technique ${ }^{9,16,17}$. The post-operative examination was carried out by a non-operative observer. On the first postoperative day, digital anteroposterior and lateral knee radiographs were completed without a strap. For the direction and inclination of the tunnel on the frontal and sagittal planes, the femoral tunnel was evaluated in radiographs. The follow-up was done weekly, then at 2-3 weeks, three months and one year. During 1-year followup, a practical performance review was conducted with the Lysholm knee scoring system. A rolimeter (Rolimeter TM for measuring anterior/posterior knee joint laxity by aircraft) was used to assess previous tibial translation quantitatively. Pivot shift testing was performed. The main objective of the study was to compare and to find out the relative progression of muscle girth readings in supervised v/s unsupervised ACL recontraction patient.

\section{Material and Method}

Eighty-two subjects (73 male 9 female) at the age group of 18-40 yrs were enrolled for this study between November 2016 to December 2018 . All the participants had undergone ACL reconstruction surgery at RML hospital Delhi. The selection of participants was volunteered and their consent for performing Ultrasound imaging was provided formally . The participants were divided into two groups as Group A: Supervised and Group B: Unsupervised. The average age of participants in supervised and unsupervised groups were 27.5 and 28.5 years respectively. The inclusion criteria were Postoperative anatomic ACL reconstruction. The exclusion criteria were any degenerative changes, bone infections, any previous injury in lower limb other than an ACL injury, any metabolic disorders like diabetes, obesity 
and hypertension, any meniscal or PCL injuryand Preoperative MRI Scan confirming ACL tear only no associated injury to menisci or other ligaments.

Assessment: Detail assessment was performed according to the assessment performa measuring the mid thigh circumfrance and special tests and strength of the muscle. WILK protocal was adopted for the strenghting exercise for both the groups for a duration of 3 months post operative.

Measurement of quadriceps muscle girth: Ultrasound is most commonly used in the assessment of soft tissue disease or fluid collection detection and may also be used to visualize other structures, such as cartilage and bone surfaces. All measurements in patients were performed three times: measurements were performed on the Kranzbüchler ultrasound machine, Medizinische Systeme GMBH, Germany, using a 7.5 MHz linear probe (Medizinische). During the measurements, the subjects lay on their backs with stretched/extended legs, their muscles relaxed and their feet in a neutral position. ${ }^{18-20}$. Both legs were examined for both the groups and muscular girth was measured by horizontal and vertical scanning of the upper leg, in the centre of the upper leg and $5 \mathrm{~cm}$ proximal and distal from the centre. In their central segments, $\mathrm{m}$. vastus intermedius and $\mathrm{m}$. rectus femoris have square shape ${ }^{21-23}$. The reproducibility of the ultrasound procedure was calculated by several measurements of 10 healthy volunteers over one day, as a change in muscle volume can not be predicted in such a short period of time. The error of this process was calculated on the basis of the variations and the uncertainty coefficients of these measurements. ${ }^{24-26}$. Measurement of thigh circumference was usually performed $15 \mathrm{~cm}$ proximal to the superior pole of the patella ${ }^{1,2,27}$. The thigh circumference was measured in $\mathrm{cm}$ with the measuring tape and compared to the normal contralateral knee to determine the amount of quadriceps atrophy present. Measurements for ultrasound scans.
Muscle dimensions were measured on off-line scans using Image J software (available http://rsb.info.nih.gov/ ij/docs/index.html). Muscle thickness was measured as the greatest vertical distance between the anterior and posterior borders of RF from their inside edges ${ }^{3,7,15}$.

Statistical tests: The SPSS 24 (Statistics System for Social Science) was used for statistical analysis. The paired t-test and was performed, which washaving a significance $\mathrm{p}$-value (less than 0.05$)^{28-30}$.

\section{Results}

The figure 2 showed the data for supervised and un-supervised groups. The following information was tabulated in the Excel spreadsheets: sex, age, ms mid quad for normal, ms mid quad in pre-operation, ms mid quad in 8 weeks of operation and ms mid quad in 12-14 weeks of operation for both supervised and unsupervised groups. A total of 164 participants were divided into two groups, i.e. 82 in each group. The pre-operative values of muscle girth for supervised was $10.83 \mathrm{~mm}$ and for unsupervised was $10.93 \mathrm{~mm}$. The ultrasound reading after 2-4 weeks post operation for supervised group was $9.83 \mathrm{~mm}$ and for un-supervised was $10.11 \mathrm{~mm}$ respectively. Both the readings of post operative 2-4 weeks, was having a downwards recovery of the muscle girth. The findings of the one pair sample statistics for supervised group is having a mean value for study group is $14.3 \mathrm{~mm}$, for normal/un-effected limb is $15.2 \mathrm{~mm}$. Whereas the standard mean difference of $0.9 \mathrm{~mm}$ for three month post operative to normal. Furthermore the standard error value of 0.24 and 0.36 for three month post operative and normal respectively. ON the other hand the values for supervised group is having a mean value for study group is $12 \mathrm{~mm}$, for normal/un-effected limb is $15 \mathrm{~mm}$. Whereas the standard mean difference of $03 \mathrm{~mm}$ for three month post operative to normal and furthermore the standard error value of 0.29 and 0.38 for three month post operative and normal respectively. 


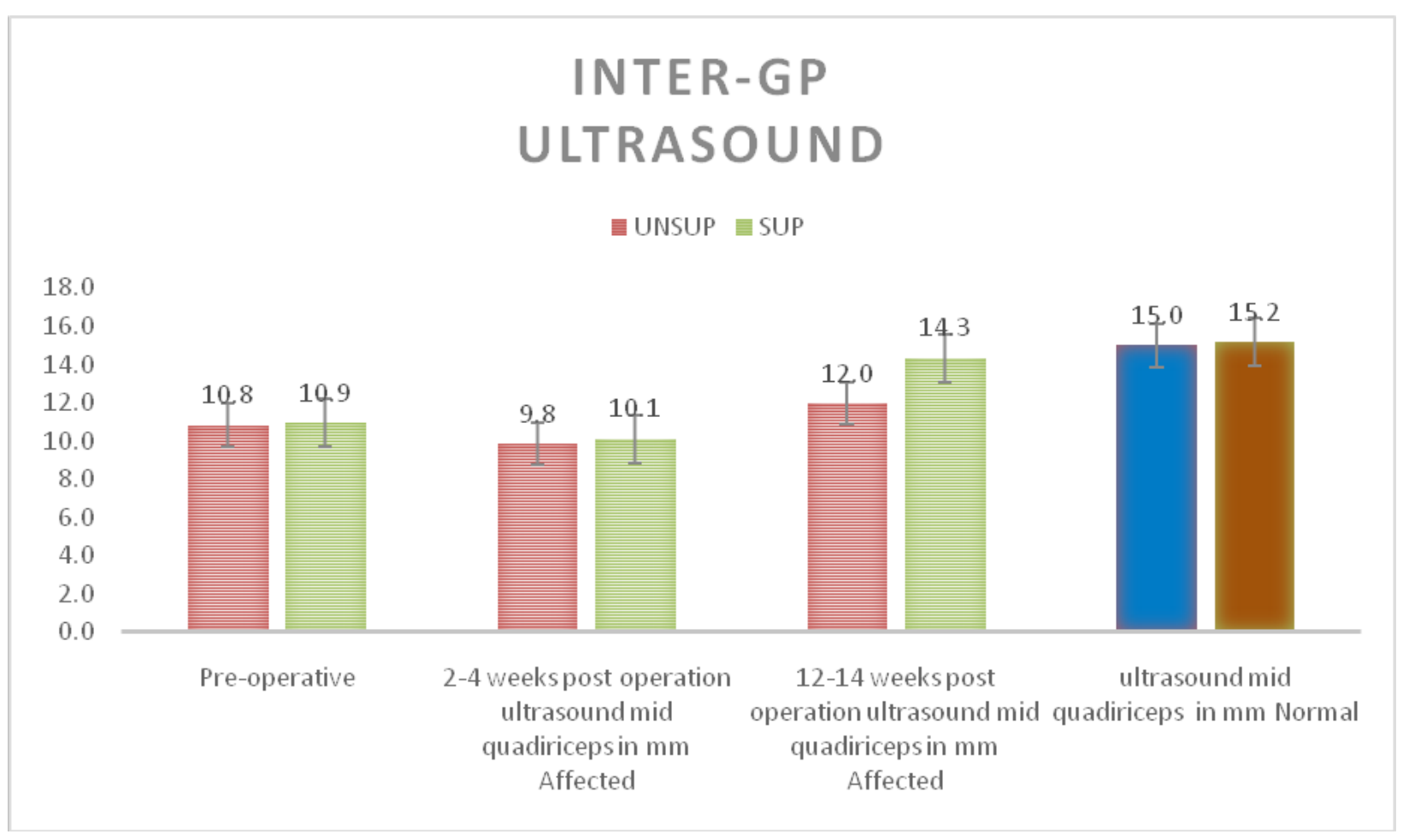

Figure 2: Supervised vs unsupervised ultrasound readings of ACL Reconstraction patients

\section{Discussion}

The ultrasound reading of the muscle girth after 2-4 weeks of post-operation showed a decline in the recovery of the muscle girth in both the groups $(-9 \%$ and $-8 \%$ for supervised and un-supervised groups). This reduction in the muscle girth value as compared to the pre-operative values was due to arthroscopic effect, which showed there was a transient deactivation of the muscle which took place due to surgery ${ }^{1}$. The ultrasound reading of the muscle girth after 12-14 weeks post-operation showed a significant progressive recovery of the muscle girth in both the groups $(31 \%$ and $10 \%$ for supervised and un-supervised groups respectively). This progression in the muscle girth value as compared to the pre-operative values (the differentce between the progression in the two group) is significant (21\%), is due to the approach in supervised exercise vs un-supervised exercise, where the focus is for isolated muscle activation of the knee. The statistical data analysis provided a comprehensive understanding of the gain in the muscle girth and the data would be inferred that the recovery rate in supervised group is relativcely approaching towards the normalicy, whereas in the un-supervised group the difference in the effected and normal is relatively higher i.e. it is deficient of recovery of muscle strength post operatively. It was also obsereved that in the um-supervised group, the muscle atrophy was very significantly visible in comparison with the supervised group. Furhtermore it was also observed that the functional strength required post operatively for the knee was lacking in the unsupervised group ${ }^{8,10,31-33}$. This study also reflected the importance and the significance of the exercise done under supervision where the physiotherapist and the patient could adopt focused approach to develop the required muscle strength and substitution of the other muscles groups could be avoided, which was lacking in the unsupervised group, where patients are complying with the exercise protocal but unable to discriminate the muscle group to be strenghted and using the gross muscles for the thigh leading to the imbalance of the muscle strength between the agonist and the antagonist group i.e. knee extensiors and knee flexures muscles ${ }^{5}$.

\section{Conclusion}

With the above study the authors concluded that survised exercises in post operative ACL reconstruction plays a very significant role for the recovery and also reduces the chances of post operative complications remarkably. 
Ethical Clearance: Taken from the ethical committee of post graduate institute of medical education \& research, Dr. Ram Monohar Lohia Hospital, New Delhi approved the study.

Source of Funding: Self funded.

\section{Conflict of Interest: Nil.}

\section{References}

1. Phelan B, King E, Richter C, Webster K, Falvey E. A comparison of anterior cruciate ligament - Return to sports after injury (ACL-RSI) scores of male athletes nine-months Post-ACL reconstruction with matched uninjured controls. Physical Therapy in Sport. 2019;38:179-183. doi:10.1016/j. ptsp.2019.05.006

2. Sinclair J, Bottoms L. Gender specific ACL loading patterns during the fencing lunge: Implications for ACL injury risk. Science and Sports. 2019;34(1):e31-e35. doi:10.1016/j. scispo.2018.05.005

3. Wilson WK, Morris R, Coskey A, Smith B, Gugala Z. Quadriceps augmentation of undersized hamstrings during ACL reconstruction. Knee. 2019;26(1):73-78. doi:10.1016/j.knee.2018.11.012

4. Yadav S, Singh S. Journal of Clinical Orthopaedics and Trauma Analysis of partial bundle anterior cruciate ligament tears- diagnosis and management with ACL augmentation. Journal of Clinical Orthopaedics and Trauma. 2019;(xxxx). doi:10.1016/j.jcot.2019.08.018

5. Rhatomy S, Hartoko L, Setyawan R, et al. Journal of Clinical Orthopaedics and Trauma Single bundle ACL reconstruction with peroneus longus tendon graft: Journal of Clinical Orthopaedics and Trauma. 2019;(xxxx):6-10. doi:10.1016/j.jcot.2019.09.004

6. Matsunaga R, Takahashi Y, Takahashi RH, et al. A new method for diagnosing biochemical abnormalities of anterior cruciate ligament (ACL) in human knees : a Raman spectroscopic study. Acta Biomaterialia. 2019. doi:10.1016/j. actbio.2019.09.016

7. Leporace G, Metsavaht L, Oliveira LP de, Nadal $\mathrm{J}$, Batista LA. Motor coordination during gait after anterior cruciate ligament injury: a systematic review of the literature. Revista Brasileira de Ortopedia (English Edition). 2013;48(4):293-299. doi:10.1016/j.rboe.2012.07.008
8. Pandy MG, Shelburne KB. Dependence of cruciateligament loading on muscle forces and external load. Journal of Biomechanics. 1997;30(10):10151024. doi:10.1016/S0021-9290(97)00070-5

9. Shelburne KB, Torry MR, Pandy MG. Muscle, ligament and joint-contact forces at the knee during walking. Medicine and Science in Sports and Exercise. 2005;37(11):1948-1956. doi:10.1249/01. mss.0000180404.86078.ff

10. Boerboom AL, Hof AL, Halbertsma JPK, et al. Atypical hamstrings electromyographic activity as a compensatory mechanism in anterior cruciate ligament deficiency. Knee Surgery, Sports Traumatology, Arthroscopy. 2001;9(4):211-216. doi:10.1007/s001670100196

11. Sharifi M, Shirazi-Adl A, Marouane H. Computational stability of human knee joint at early stance in Gait: Effects of muscle coactivity and anterior cruciate ligament deficiency. Journal of Biomechanics. 2017;63:110-116. doi:10.1016/j. jbiomech.2017.08.004

12. Branch TP, Hunter R, Donath M. Dynamic EMG analysis of anterior cruciate deficient legs with and without bracing during cutting. The American Journal of Sports Medicine. 1989;17(1):35-41. doi:10.1177/036354658901700106

13. Hasegawa S, Kobayashi M, Arai R, Tamaki A, Nakamura T, Moritani T. Effect of early implementation of electrical muscle stimulation to prevent muscle atrophy and weakness in patients after anterior cruciate ligament reconstruction. Journal of Electromyography and Kinesiology. 2011;21(4):622-630. doi:10.1016/j. jelekin.2011.01.005

14. Mothersill C, Seymour CB, O'Brien A. Induction of c-myc oncoprotein and of cellular proliferation by radiation in normal human urothelial cultures. Anticancer Research. 1991;11(4):1609-1612. doi:10.1002/jor

15. Bennett MJ, Billing S, Hollar AC, Roe A. Strength Response in Human Quadriceps. Journal of Orthopaedic \& Sports Physical Therapy. 2003;2.

16. Lass $\mathrm{P}$, Kaalund S, Iefevre S, Arendt-Nielsen L, Sinkjæ T, Simonsen O. Muscle coordination following rupture of the anterior cruciate ligament: Electromyographic studies of 14 patients. Acta Orthopaedica. 1991;62(1):9-14. doi:10.3109/17453679108993083 
17. Horton MG, Hall TL. Quadriceps Femoris Muscle Angle : Normal Values and Relationships with Gender and Selected Skeletal Measures. Physical therapy. 1989;69(11):897-901. doi:10.1093/ $\mathrm{ptj} / 69.11 .897$

18. Giles LS, Webster KE, McClelland JA, Cook J. Can ultrasound measurements of muscle thickness be used to measure the size of individual quadriceps muscles in people with patellofemoral pain? Physical Therapy in Sport. 2015;16(1):45-52. doi:10.1016/j.ptsp.2014.04.002

19. Tuite MJ, DeSmet AA. MRI of selected sports injuries: Muscle tears, groin pain and osteochondritis dissecans. Seminars in Ultrasound, CT and MRI. 1994;15(5):318-340. doi:10.1016/ S0887-2171(05)80002-2

20. Bemben DA, Langdon DB. Relationship between estrogen use and musculoskeletal function in postmenopausal women. Maturitas. 2002;42(2):119-127. doi:10.1016/S03785122(02)00033-6

21. Tang ZW, He Y, Yao Y, Qiu L, Tian HM. Size of quadriceps femoris may contribute to thyrotoxic periodic paralysis. Medical Hypotheses. 2015;85(6):749-753. doi:10.1016/j. mehy.2015.10.014

22. Wong LLS. Imaging of muscle injuries. Journal of the Hong Kong College of Radiologists. 2005;8(4):191-201.

23. Thijssen DH, Ellenkamp R, Smits P, Hopman MT. Rapid vascular adaptations to training and detraining in persons with spinal cord injury. Archives of Physical Medicine and Rehabilitation. 2006;87(4):474-481. doi:10.1016/j. apmr.2005.11.005

24. Adeoye O, Christianah O, Spirina G, Cui D, Lynch JC, Lehman MN. ISMS2017-1-001 Radiofrequency electromagnetic radiation from cell phone causes defective testicular function in male ISMS20171-003 Comparative study of the ear of black and white ISMS2017-1-002 Influence of variations of coronary artery branches on blood. Annals of Anatomy - Anatomischer Anzeiger. 2017;1:6-334. doi:10.1016/j.aanat.2017.05.003

25. Vanlaningham E. American Association of Electrodiagnostic Medicine, 39th annual meeting.
Charleston, South Carolina, 16-17 October 1992. Abstracts. Electroencephalography and Clinical Neurophysiology. 1993;87(4).

26. Williams SK, Rabbani F. Complications of Lymphadenectomy in Urologic Surgery. Urologic Clinics of North America. 2011;38(4):507-518. doi:10.1016/j.ucl.2011.07.013

27. BREIDAHL P. The Use of Computed Tomography in Assessing Muscle Cross-sectional Area and the Relationship between Cross-sectional Area and Strength. Australian Journal of Physiotherapy. 1987;33(2):75-82. doi: $10.1016 / \mathrm{S} 0004-$ 9514(14)60585-7

28. Dixit S, Mandal SN, Thanikal J V, Saurabh K. Evolution of studies in construction productivity: A systematic literature review (2006-2017). Ain Shams Engineering Journal. 2019. doi:10.1016/j. asej.2018.10.010

29. Dixit S, Sharma K. An Empirical Study of Major Factors Affecting Productivity of Construction Projects. In: Babu KG, Rao HS, Amarnath Y, eds. Emerging Trends in Civil Engineering. Singapore: Springer Singapore; 2020:121-129.

30. Dixit S, Sharma K, Singh S. Identifying and Analysing Key Factors Associated with Risks in Construction Projects. In: Babu KG, Rao HS, Amarnath Y, eds. Emerging Trends in Civil Engineering. Singapore: Springer Singapore; 2020:25-32.

31. June T. World Physical Therapy 2007 - Abstracts. Physiotherapy. 2007;93:S1-S802. doi:10.1016/ s0031-9406(07)60001-7

32. Sinha S, Naik AK, Maheshwari M, Sandanshiv S, Meena D. Anterior Cruciate Ligament Reconstruction with Tibial Attachment Preserving Hamstring Graft without Implant on Tibial Side. 2018;52(2):170-176. doi:10.4103/ortho.IJOrtho

33. Ciccotti MG, Kerlan RK, Perry J, Pink M. An Electromyographic Analysis of the Knee During Functional Activities 2: the Anterior Cruciate Ligament-Deficient and LigamentReconstructed Profiles. American Journal of Sports Medicine. 1994;22(5):651-658. doi:10.1177/036354659402200513 